BULL. AUSTRAL. MATH. SOC.

VOL. $29(1984), 259-265$.

\title{
LOCALLY UNIFORMLY ROTUND RENORMING AND DECOMPOSITIONS OF BANACH SPACES
}

\author{
V. ZIZLER
}

\begin{abstract}
A norm $|\cdot|$ of a Banach space $X$ is called locally uniformly rotund if $\lim \left|x_{n}-x\right|=0$ whenever $x_{n}, x \in X$, and $\lim 2|x|^{2}+2\left|x_{n}\right|^{2}-\left|x+x_{n}\right|^{2}=0$. It is shown that such an equivalent norm exists on every Banach space $X$ which possesses a projectional resolution $\left\{P_{\alpha}\right\}$ of the identity operator, for which all $\left(P_{\alpha+1}-P_{\alpha}\right) X$ admit such norms. This applies, for example, for the dual space of a space with Fréchet differentiable norm.
\end{abstract}

Projectional resolutions of the identity in nonseparable Banach spaces were first studied by Amir and Lindenstrauss [1]. It has proved to be a very powerful tool in the study of geometry of some nonseparable Banach spaces. For example, it was used to prove that every weakly compactly generated Banach space admits an equivalent locally uniformly rotund norm in [6], and that the dual space of a space with Fréchet smooth norm admits an equivalent strictly convex norm in [4]. In the projectional resolutions used in [6], the spaces $\left(P_{\alpha+1}-P_{\alpha}\right) X$ were all separable. Sometimes one

Received 31 October 1983. The author is deeply indebted to the Sonderforschungsbereich 72 der Universität Bonn for the support during the preparation of the paper. He also thanks the University of Dortmund for providing him with excellent working conditions during his stay there.

Copyright Clearance Centre, Inc. Serial-fee code: 0004-9727/84 $\$ A 2.00+0.00$. 
cannot ensure this separability requirement, but only the fact that $\left(P_{\alpha+1}{ }^{-P_{\alpha}}\right) X$ all admit equivalent locally uniformly rotund norms. Then, a slight variant of the construction from [6], given here, works to give the result. This is, for example, the case mentioned in the abstract.

We will work in real Banach spaces. $N$ will denote the set of all positive integers.

THEOREM 1. Let $(X,|\cdot|)$ be a Bconach space which possesses a farily $\left\{P_{\alpha}\right\}, \quad \alpha \in \Gamma$, of bounded Zinear operators $P_{\alpha}: X \rightarrow X$, such that

(i) the map $T$ defined on $X$ by $T x(\gamma)=\left|P_{\gamma} x\right|$ for $x \in X$, maps $X$ into $c_{0}(\Gamma)$,

(ii) if $x \in X$, then $x \in \overline{\operatorname{sp}}\left\{P_{\alpha} x\right\}$,

(iii) for each $\alpha \in \Gamma, P_{\alpha} X$ admits an equivalent locally uniformly rotund norm.

Then $X$ admits an equivalent locally uniformly rotund norm.

An application of Theorem 1 is given below.

COROLLARY 1. Suppose that $X$ is a Banach space which admits a real valued continuously Fréchet differentiable fronction with bounded non-empty support. Then $X^{*}$ admits an equivalent locally uniformly rotund norm.

Proof. Using the method of [5], it was shown in [4] that under our assumption on $X$, the identity operator on $X^{*}$ admits a projectional resolution $\left\{P_{\alpha}\right\}$ for which each $P_{\alpha} X^{*}$ is isometric to some $X^{*}$ where dens $X_{\alpha}=$ dens $X_{\alpha}^{*}<$ dens $X=$ dens $X^{*}$. So, using a transfinite induction argument on dens $X$, one can ensure the existence of operators needed in Theorem 1 , by the projections $\left(P_{\alpha+1}-P_{\alpha}\right)$ on $X^{*}$. This proves Corollary 1 from Theorem 1 .

Proof of Theorem 1. A variant of that in [6]. Assume, without loss of generality, that $\left|P_{\alpha}\right| \leq 1, \alpha \in \Gamma$. Let $h_{\alpha}(x)=\left|P_{\alpha} x\right|_{\alpha}$ where $\alpha \in \Gamma$ and $x \in X$, and where $\left.1 \cdot\right|_{\alpha}$ is an equivalent locally uniformly rotund norm on $P_{\alpha} X$ such that $|\cdot| \leq|\cdot|_{\alpha} \leq 2|\cdot|$. Furthermore, for $k \in N$, let 
$r_{j}^{k}=\left(r_{j, 1}^{k}, \ldots, r_{j, k}^{k}\right), j=1,2, \ldots$ be a sequence of all (ordered) $k$-tuples of rational numbers. Let $Q$ be a map which assigns to each finite subset $A \subset \Gamma$ an enumeration of $A$ to an (ordered) sequence $Q(A)=\left(\alpha_{1}, \ldots, \alpha_{n}\right), \alpha_{i}$ distinct.

If $A \subset \Gamma$, where card $A=n$ and $Q(A)=\left(\alpha_{1}, \ldots, \alpha_{n}\right)$ and if $j, Z \in N$ define

$$
E(A, j, i)(x)=\left(\sum_{\alpha \in A} h_{\alpha}^{2}(x)+\frac{1}{2} \cdot 1 /\left(\sum_{i=1}^{n}\left|r_{j, i}^{n}\right|+1\right)^{2}\left|x-\sum_{i=1}^{n} r_{j, i}^{n} P_{\alpha_{i}} x\right|^{2}\right)^{\frac{1}{2}}
$$

for $x \in X$. Furthermore, if $j, \imath, n \in N$ are fixed, let

$$
G(j, Z, n)(x)=\sup \{E(A, j, Z)(x), A \subset \Gamma, \operatorname{card} A=n\}
$$

for $x \in X$.

Finally, let

$$
\|x\|=\left(|x|^{2}+\sum \frac{1}{2^{j+z+n}} G^{2}(j, z, n)(x)\right)^{\frac{1}{2}}
$$

for $x \in X$. Then it is easy to see that $\|\cdot\|$ is an equivalent norm on $X$.

We shall show that it is locally uniformly rotund.

To this end suppose $x_{k}, x \in X$, are such that $|x|=1$ and

$$
\lim 2\|x\|^{2}+2\left\|x_{k}\right\|^{2}-\left\|x+x_{k}\right\|^{2}=0
$$

We need show that $\lim \left|x_{k}-x\right|=0$. Thus, given $\varepsilon>0$, we shall show that beginning with some index $k_{0},\left|x_{k}-x\right|<4 \varepsilon$. For that, first find a set $A \subset\left\{\alpha \in \Gamma, h_{\alpha}(x) \neq 0\right\}, Q(A)=\left(\alpha_{1}, \ldots, \alpha_{n}\right\}$ such that $\rho\left(x, \operatorname{sp}\left\{P_{\alpha} x, \alpha \in A\right\}\right)<\varepsilon$.

Assume without loss of generality that

$$
\min _{\alpha \in A} h_{\alpha}^{2}(x)-\max _{\alpha \notin A} h^{2}(x)=d>0 .
$$

Let 
262

V. Zizler

(2)

$$
\left|x-\sum_{s=1}^{n} r_{s} P_{\alpha_{s}} x\right|<\varepsilon
$$

where $\left(r_{1}, \ldots, r_{n}\right)$ is a sequence of rationals.

Choose $j \in N$ so that $r_{j}^{n}=\left(r_{j, 1}^{n}, \ldots, r_{j, n}^{n}\right)=\left(r_{1}, \ldots, r_{n}\right)$.

Finally let $l>4 / d$. Fix these $n, j, l$. From (l) we have that

(3) $a_{k}=2 G^{2}(j, \imath, n)(x)+2 G^{2}(j, l, n)\left(x_{k}\right)-G^{2}(j, l, n)\left(x+x_{k}\right) \underset{k}{\longrightarrow} 0$.

Let $A_{k} \subset \Gamma, \quad \operatorname{card} A_{k}=n, Q\left(A_{k}\right)=\left(\alpha_{1}^{k}, \ldots, \alpha_{n}^{k}\right)$, be so that

(4)

$$
0 \leq c_{k}=G^{2}(j, \imath, n)\left(x+x_{k}\right)-E^{2}\left(A_{k}, j, \imath\right)\left(x+x_{k}\right) \underset{k}{\longrightarrow} 0 .
$$

Then

$$
\begin{aligned}
a_{k} & \geq 2 E^{2}\left(A_{k}, j, l\right)(x)+2 E^{2}\left(A_{k}, j, l\right)\left(x_{k}\right)-E^{2}\left(A_{k}, j, l\right)\left(x+x_{k}\right)-c_{k} \\
& =b_{k}-c_{k}
\end{aligned}
$$

for some nonnegative $b_{k}$ and thus, since $\lim a_{k}=\lim c_{k}=0$, we have that $\lim b_{k}=0$ as well. Thus

$$
\begin{aligned}
& b_{k}=2 \sum_{\alpha \in A_{k}} h_{\alpha}^{2}(x)+\frac{2}{2}\left(1 /\left(\sum_{i=1}^{n}\left|r_{j, i}^{n}\right|+1\right)^{2}\right)\left|x-\sum_{i=1}^{n} r_{j, i}^{n} \alpha_{i}^{k^{x}}\right|^{2} \\
& +2 \sum_{\alpha \in A_{k}} h_{\alpha}^{2}\left(x_{k}\right)+\frac{2}{2}\left(1 /\left(\sum_{i=1}^{n}\left|r_{j, i}^{n}\right|+1\right)^{2}\right)\left|x_{k}-\sum_{i=1}^{n} r_{j, i}^{n} \alpha_{i}^{k_{k} x_{k}}\right|^{2} \\
& -\sum_{\alpha \in A_{k}} h_{\alpha}^{2}\left(x+x_{k}\right) \\
& -\frac{1}{2} \cdot 1 /\left(\sum_{i=1}^{n}\left|r_{j, i}^{n}\right|+1\right)^{2}\left|x+x_{k}-\sum_{i=1}^{n} r_{j, i}^{n}{ }_{\alpha_{i}^{k}}\left(x+x_{k}\right)\right|^{2} \underset{k}{\longrightarrow} 0 .
\end{aligned}
$$

Thus by the convexity argument,

$$
2 \sum_{\alpha \in A_{k}} h_{\alpha}^{2}(x)+2 \sum_{\alpha \in A_{k}} h_{\alpha}^{2}\left(x_{k}\right)-\sum_{\alpha \in A_{k}} h_{\alpha}^{2}\left(x+x_{k}\right) \underset{k}{\longrightarrow} 0
$$

https://doi.org/10.1017/S0004972700021493 Published online by Cambridge University Press 
and

$$
\left|x-\sum_{i=1}^{n} r_{j, i}^{n}{ }_{\alpha_{i}^{k}}{ }^{x}\right|-\left|x_{k}-\sum_{i=1}^{n} r_{j, i}^{n}{ }_{\alpha_{i}^{k}}^{x_{k}}\right|+0 .
$$

We now show that, beginning with some index $k_{1}, A_{k}=A$ and so then $\left(\alpha_{1}^{k}, \ldots, \alpha_{n}^{k}\right)=\left(\alpha_{1}, \ldots, \alpha_{n}\right)$. Indeed, if this were not the case, we would have for infinitely many $k, A_{k} \neq A$. But for these $k$ we would have

$$
\begin{aligned}
& a_{k} \geq 2 \sum_{\alpha \in A} h^{2}(x)+\frac{2}{2}\left(1 /\left(\sum_{i=1}^{n}\left|r_{j, i}^{n}\right|+1\right)^{2}\right)\left|x-\sum_{i=1}^{n} r_{j, i}^{n} P_{\alpha_{i}} x\right|^{2}+2 \sum_{\alpha \in A_{k}} h^{2}\left(x_{k}\right) \\
& +\frac{2}{2}\left(I /\left(\sum_{i=1}^{n}\left|r_{j, i}^{n}\right|+1\right)^{2}\right)\left|x_{k}-\sum_{i=1}^{n} r_{j, i}^{n}{ }_{\alpha}{ }_{k}^{k} x_{k}\right|^{2}-\sum_{\alpha \in A_{k}} h_{\alpha}^{2}\left(x+x_{k}\right) \\
& -\frac{1}{2}\left(1 /\left(\sum_{i=1}^{n}\left|r_{j, i}^{n}\right|+1\right)^{2}\right)\left|x+x_{k}-\sum_{i=1}^{n} r_{j, i}^{n} \alpha_{i}^{k}\left(x+x_{k}\right)\right|^{2}-c_{k} \\
& =2\left(\sum_{\alpha \in A} h_{\alpha}^{2}(x)-\sum_{\alpha \in A_{k}} h_{\alpha}^{2}(x)\right) \\
& +\frac{2}{2}\left(I /\left(\sum_{i=1}^{n}\left|r_{j, i}^{n}\right|+1\right)^{2}\right)\left(\left|x-\sum_{i=1}^{n} r_{j, i}^{n} P_{\alpha_{i}} x\right|^{2}-\left|x-\sum_{i=1}^{n} r_{j, i}^{n}{ }_{\alpha_{i}^{k}}^{x}\right|^{2}\right) \\
& +2 \sum_{\alpha \in A_{k}} h_{\alpha}^{2}(x)+\frac{2}{2}\left(1 /\left(\sum_{i=1}^{n}\left|r_{j, i}^{n}\right|+1\right)^{2}\right)\left|x-\sum_{i=1}^{n}{ }_{j, i}^{n} \alpha_{i} k^{x}\right|^{2}
\end{aligned}
$$

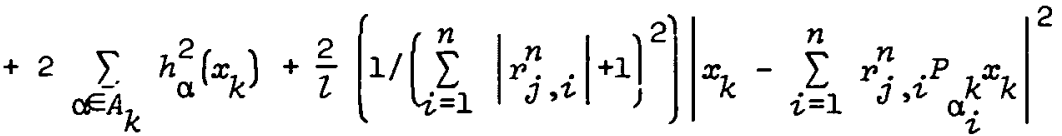

$$
\begin{aligned}
& -\sum_{\alpha \in A_{k}} h_{\alpha}^{2}\left(x+x_{k}\right)-\frac{1}{2}\left(1 /\left(\sum_{i=1}^{n}\left|r_{j, i}^{n}\right|+1\right)^{2}\right)\left|x+x_{k}-\sum r_{j, i}^{n}{ }_{\alpha_{i}^{k}}\left(x+x_{k}\right)\right|^{2}-c_{k} \\
& \geq 2 d-\frac{4}{2}-c_{k} \geq d-c_{k} \text {, }
\end{aligned}
$$

which is a contradiction with the fact that $\lim a_{k}=\lim c_{k}=0, d>0$. 
Therefore, beginning with some index $k_{1}$, we must have that $A_{k}=A$ and thus $\left(\alpha_{1}^{k}, \ldots, \alpha_{n}^{k}\right)=\left(\alpha_{1}, \ldots, \alpha_{n}\right)$.

Now from (5) we have that, for each $\alpha \in A$,

$$
2 h_{\alpha}^{2}(x)+2 h_{\alpha}^{2}\left(x_{k}\right)-h_{\alpha}^{2}\left(x+x_{k}\right) \underset{k}{\longrightarrow} 0
$$

and thus from the locally uniformly rotund of $|\cdot|_{\alpha}$, we have that $P_{\alpha}\left(x_{k}-x\right) \underset{k}{\longrightarrow} 0$. Therefore

$$
\left|\sum_{i=1}^{n} r_{j, i}^{n}{ }_{\alpha_{i}} x-\sum_{i=1}^{n} r_{j, i}^{n}{ }_{\alpha_{i}} x_{k}\right| \underset{k}{\longrightarrow} 0
$$

and using this and (2) and (6) we have that beginning with some index $k_{0}$, we have that $\left|x-x_{k}\right|<4 \varepsilon$. The proof is finished.

REMARK 1. A version of Corollaxy 1 was published in [2]. However, we are afraid that the proof given in [2] is not correct.

REMARK 2. The locally uniformly rotund norm constructed in Corollary 1 on $X^{*}$ cannot generally be made to be a dual one (see [5]).

\section{References}

[1] D. Amir and J. Lindenstrauss, "The structure of weakly compact sets in Banach spaces", Ann. of Math. (2) 88 (1968), 35-46.

[2] C.M. Гутман [S.M. Gutman], "Об эквивалентных нормах в некоторых несепарабедыных В-пространствах" [Equivalent norms in certain nonseparable B-spaces], Teor. Funkcii Funkcional. Anal. $i$ Prizožen. 20 (1974), 63-69.

[3] K. John and V. Zizler, "Duals of Banach spaces which admit nontrivial smooth functions", Bull. Austral. Math. Soc. 11 (1974), 161-166.

[4] D.G. Tacon, "The conjugate of a smooth Banach space", Bulz. Austral. Math. Soc. 2 (1970), 415-425.

[5] Michel Talagrand, "Renormages de quelques $\mathrm{C}(\mathrm{K})$ ", to appear. 
[6] S. Troyanski, "On locally uniformly convex and differentiable norms in certain non-separable Banach spaces", Studia Math. 37 (1971), 173-180.

Institut fur Angewandte Mathematik der Universitat Bonn, Wegelerstr. 6,

D-5300 Bonn,

West Germany

and

Department of Mathematics,

University of Alberta,

Edmonton T6G 2GI,

Alberta,

Canada. 